\title{
Article \\ Cytotoxic Indole-Diterpenoids from the Marine-Derived Fungus Penicillium sp. KFD28
}

\author{
Lu-Ting Dai ${ }^{1}{ }^{(D}$, Li Yang ${ }^{2}$, Fan-Dong Kong ${ }^{3}$, Qing-Yun Ma ${ }^{2}$, Qing-Yi Xie ${ }^{2}$, Hao-Fu Dai ${ }^{4}$, Zhi-Fang Yu ${ }^{1, *}$ \\ and You-Xing Zhao ${ }^{2, *}$
}

1 College of Food Science and Technology, Nanjing Agricultural University, Nanjing 210095, China; dailuting121@163.com

2 Haikou Key Laboratory for Research and Utilization of Tropical Natural Products, Institute of Tropical Bioscience and Biotechnology, CATAS, Haikou 571101, China; yangli@itbb.org.cn (L.Y.); maqingyun@itbb.org.cn (Q.-Y.M.); xieqingyi@itbb.org.cn (Q.-Y.X.)

3 Key Laboratory of Chemistry and Engineering of Forest Products, State Ethnic Affairs Commission, Guangxi Key Laboratory of Chemistry and Engineering of Forest Products, Guangxi Collaborative Innovation Center for Chemistry and Engineering of Forest Products, School of Chemistry and Chemical Engineering, Guangxi University for Nationalities, Nanning 530006, China; kongfandong501@126.com

4 Hainan Institute for Tropical Agricultural Resources, CATAS, Haikou 571101, China; daihaofu@itbb.org.cn

* Correspondence: yuzhifang@njau.edu.cn (Z.-F.Y.); zhaoyouxing@itbb.org.cn (Y.-X.Z.); Tel.: +86-139-5169-2350 (Z.-F.Y.); +86-898-6698-9095 (Y.-X.Z.)

check for updates

Citation: Dai, L.-T.; Yang, L.; Kong, F.-D.; Ma, Q.-Y.; Xie, Q.-Y.; Dai, H.-F.; Yu, Z.-F.; Zhao, Y.-X. Cytotoxic Indole-Diterpenoids from the Marine-Derived Fungus Penicillium sp. KFD28. Mar. Drugs 2021, 19, 613. https://doi.org/10.3390/md19110613

Academic Editor: Khaled A. Shaaban

Received: 28 September 2021

Accepted: 24 October 2021

Published: 28 October 2021

Publisher's Note: MDPI stays neutral with regard to jurisdictional claims in published maps and institutional affiliations.

Copyright: (c) 2021 by the authors. Licensee MDPI, Basel, Switzerland. This article is an open access article distributed under the terms and conditions of the Creative Commons Attribution (CC BY) license (https:/ / creativecommons.org/licenses/by/ $4.0 /)$.

\begin{abstract}
Four new indole-diterpenoids, named penerpenes K-N (1-4), along with twelve known ones (5-16), were isolated from the fermentation broth produced by adding L-tryptophan to the culture medium of the marine-derived fungus Penicillium sp. KFD28. The structures of the new compounds were elucidated extensively by 1D and 2D NMR, HRESIMS data spectroscopic analyses and ECD calculations. Compound 4 represents the second example of paxilline-type indole diterpene bearing a 1,3-dioxepane ring. Three compounds $(4,9$, and 15$)$ were cytotoxic to cancer cell lines, of which compound 9 was the most active and showed cytotoxic activity against the human liver cancer cell line BeL-7402 with an $\mathrm{IC}_{50}$ value of $5.3 \mu \mathrm{M}$. Moreover, six compounds $(5,7,10,12,14$, and 15) showed antibacterial activities against Staphylococcus aureus ATCC 6538 and Bacillus subtilis ATCC 6633.
\end{abstract}

Keywords: marine-derived fungus; Penicillium sp.; indole-diterpenoids; cytotoxicity; antibacterial activity

\section{Introduction}

Marine fungi have formed different metabolic pathways and adaptation mechanisms within the peculiar marine environment. Hence, marine fungi can produce natural secondary metabolites that are characterized by unique chemical structures and high biological activities [1,2]. Alkaloids derived from marine-derived compounds have received extensive attention in recent years. Indole alkaloids, as an important class of secondary metabolites produced by marine-derived fungi [3], showed excellent biological activities, including cytotoxic [4], antibacterial [5], quorum sensing inhibitory [6], anti-Zika virus [7], and protein tyrosine phosphatase inhibitory activities [8,9].

Marine-derived fungus Penicillium sp. KFD28 was isolated and identified from bivalve shellfish, Meretrix lusoria, collected from Haikou Bay, China. Our previous study on the secondary metabolites of this fungus discovered a series of indole alkaloids with novel structures and intriguing bioactivities, e.g., protein tyrosine phosphatase inhibitory activity [8,9]. The OSMAC (one strain, many compounds) approach is highly efficient for inducing structural diversity by the variation of cultivation conditions [10]. Most of the indole alkaloids precursors are related to L-tryptophan [11]. To find more new alkaloids from marine fungi, adding amino acids to the culture media is becoming a viable 
strategy $[12,13]$. In order to explore the metabolic potential of the fungus Penicillium sp. KFD28, we recultured this fungus by adding L-tryptophan to the solid rice culture and found that the HPLC profiles of the extract are different from that of the first time obtained from the liquid medium. Later chemical investigation of the fermentation broth led to the isolation of four new indole-diterpenoids, penpenes K-N (1-4), along with 12 known ones, including paxilline (5) [14], dehydroxypaxilline (6) [15], 7-hydroxyl-13-dehydroxypaxilline (7) [16], 3-deoxo-4b-deoxypaxilline (8) [17], epipaxilline (9) [18], 7-hydroxypaxilline-13-ene (10) [19], paspaline (11) [20], 4a-demethylpaspaline-4a-carboxylic acid (12) [17], paspalinine (13) [21], PC-M6 (14) [22], emindole SB (15) [23], emeniveol (16) [24] (Figure 1). Herein, the isolation, structure elucidation, and bioactivities of these compounds are reported.

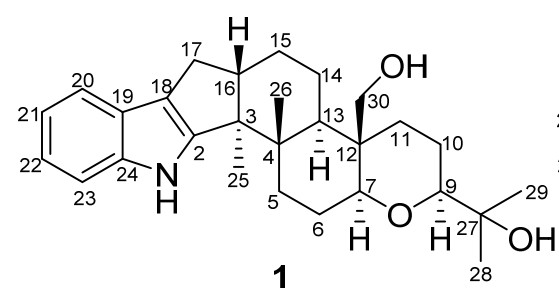<smiles>CC(C)(O)[C@@H]1OC(=O)C=C2[C@H](CC[C@]3(C)[C@H]2CC[C@H]2Cc4c([nH]c5ccccc45)[C@@]23C)O1</smiles>
${ }_{29} \mathrm{OH}$

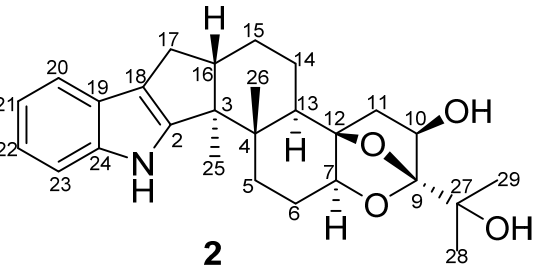

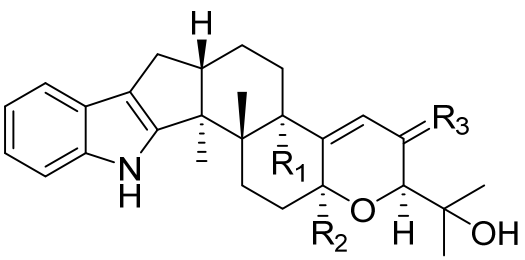

$5 \mathrm{R}_{1}=\mathrm{OH} \quad \mathrm{R}_{2}=\mathrm{H} \mathrm{R}_{3}=\mathrm{O}$

$6 \mathrm{R}_{1}=\mathrm{R}_{2}=\mathrm{H} \mathrm{R}_{3}=\mathrm{O}$

$7 \mathrm{R}_{1}=\mathrm{H} \mathrm{R}_{2}=\mathrm{OH} \mathrm{R} \mathrm{R}_{3}=\mathrm{O}$

$8 \mathrm{R}_{1}=\mathrm{R}_{2}=\mathrm{H} \mathrm{R}_{3}=\mathrm{H}_{2}$

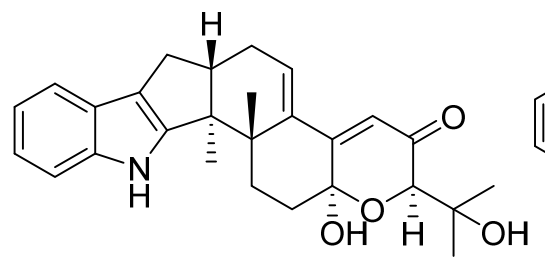

10<smiles>[R1][C@]12CC[C@H](C(C)(C)O)O[C@H]1CC[C@@]1(C)[C@@H]2CC[C@H]2Cc3c([nH]c4ccccc34)[C@@]21C</smiles>

$11 \mathrm{R}_{1}=\mathrm{CH}_{3}$

$12 \mathrm{R}_{1}=\mathrm{COOH}$

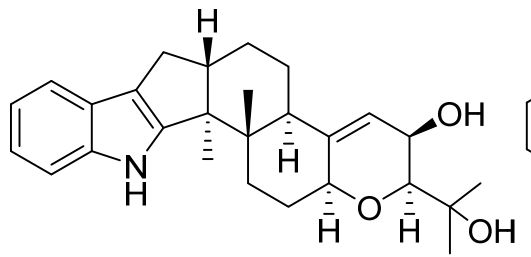

14<smiles>CC(C)=CCC[C@]1(C)C2CC[C@H](O)[C@@H](O)CC2[C@@]2(C)Cc3c([nH]c4ccccc34)[C@@H]12</smiles>

15

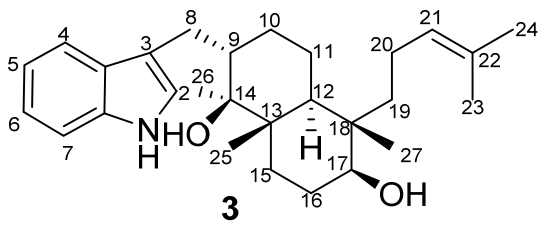<smiles>CC(C)(O)[C@]1(C)O[C@H]2CC[C@]3(C)[C@@]4(C)c5[nH]c6ccccc6c5C[C@H]4CC[C@]3(O)C2=CC1=O</smiles>

9

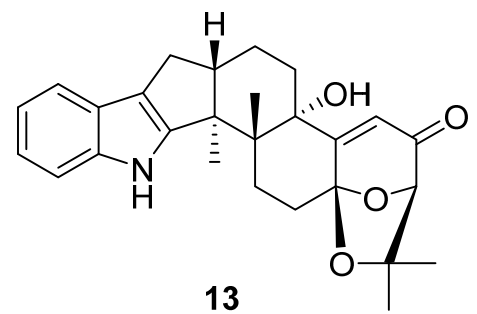

13<smiles>C=C1[C@H](Cc2c[nH]c3ccccc23)CCC2[C@]1(C)CC[C@@H](O)[C@]2(C)CCC=C(C)C</smiles>

16

Figure 1. The chemical structures of compounds 1-16.

\section{Results and Discussions}

\subsection{Structure Elucidation}

Compound 1 was obtained as a yellow oil. Its formula was determined as $\mathrm{C}_{28} \mathrm{H}_{39} \mathrm{NO}_{3}$ on the basis of HRESIMS data $\left(m / z 476.2565\right.$ for $\left.[\mathrm{M}+\mathrm{K}]^{+}\right)$, indicating ten degrees of unsaturation. The ${ }^{1} \mathrm{H}$ and ${ }^{13} \mathrm{C}$ NMR data of 1 , with the aid of its HSQC spectrum (Supplementary Materials, Figures S1-S4), showed a total of 28 carbon signals comprising eight olefinic 
or aromatic carbons (four protonated) for a 2,3-disubstituted indole moiety, four methyls, eight $\mathrm{sp}^{3}$ methylenes with one oxygenated, four $\mathrm{sp}^{3}$ methines with two oxygenated, and four non-protonated $\mathrm{sp}^{3}$ carbons with one oxygenated. These NMR data allowed the construction of the carbon skeleton of indole-diterpenoid. Careful contrast of the similar NMR spectral data between 1 and paspaline (11) [20] revealed that they had the same planar structure except that the methyl group at C-12 $\left(\delta_{\mathrm{C}} 36.6\right)$ in paspaline was oxidized to oxymethylene in 1. Consistent with the introduction of the hydroxyl at C-30 $\left(\delta_{\mathrm{C}} 58.5\right)$, it showed a distinctive deshielding of the C-12 signal $\left(\delta_{\mathrm{C}} 48.8\right)$ in 1 compared to those of paspaline $\left(\delta_{\mathrm{C}} 36.6\right)$. The linkage of a hydroxyl at C-30 was further supported by the COSY correlation of 30-OH/H -30 (Figure 2; Supplementary Material, Figure S8) and ROESY correlation of $\mathrm{H}_{3}-26 / \mathrm{H}_{2}-30$ (Supplementary Material, Figure S9). Detailed analysis of 2D NMR data further confirmed that $\mathbf{1}$ and paspaline share the same indole-diterpenoid skeleton. The relative configuration of 6/6/6 tricyclic rings in indole-diterpenoid were determined by the ROESY spectrum (Figure 3), in which the sequential correlations of $\mathrm{H}-16 / \mathrm{H}_{3}-26 / \mathrm{H}_{2}-$ $30 / \mathrm{H}-10 \beta / \mathrm{H}_{3}-28$ suggested the same face of $\mathrm{H}-16, \mathrm{CH}_{3}-26, \mathrm{CH}_{2}-30$, and $\mathrm{C}-27$ in the $6 / 6 / 6$ tricyclic ring system, while the correlations of $\mathrm{H}_{3}-25 / \mathrm{H}-13 / \mathrm{H}-7 / \mathrm{H}-9$ suggested $\mathrm{CH}_{3}-25$, $\mathrm{H}-13, \mathrm{H}-7$, and H-9 were on the opposite face of this system. It has been reported that the strong Cotton effect (CE) around $220 \mathrm{~nm}$ was related to the absolute configurations of the chiral carbons around the indole chromophore in the paxilline-type indole-diterpene [17]. Thus, the strong negative CE at $223 \mathrm{~nm}$ in the experimental ECD spectrum of $\mathbf{1}$ (Figure 4) suggested its $(3 S, 4 S, 7 S, 9 S, 12 S, 13 R, 16 S)-\mathbf{1}$ absolute configuration [17]. As for the absolute configuration of $\mathbf{1}$, the ECD spectrum of $(3 S, 4 S, 7 S, 9 S, 12 S, 13 R, 16 S)-1$ was calculated; this deduction was further supported, establishing the absolute configuration of $\mathbf{1}$ as presented in Figure 1.

Compound 2 was isolated as a yellow oil. Its formula was determined as $\mathrm{C}_{27} \mathrm{H}_{35} \mathrm{NO}_{4}$ on the basis of HRESIMS data, indicating 11 degrees of unsaturation. Its ${ }^{13} \mathrm{C}$ NMR data showed a total of 27 carbon signals comprising eight aromatic or olefinic carbons for an indole moiety, four methyls, six $\mathrm{sp}^{3}$ methylenes, four $\mathrm{sp}^{3}$ methines with two oxygenated, and five non-protonated $\mathrm{sp}^{3}$ carbons with three oxygenated. Analysis of the NMR spectra (Tables 1 and 2, Supplementary Materials, Figures S12-S18) of 2 suggested that its structure was related to that of 4a-demethylpaspaline-3,4,4a-triol [17], and the main difference being C-9 $\left(\delta_{C} 109.8\right), C-11\left(\delta_{C} 43.7\right)$, and C-12 $\left(\delta_{C} 84.6\right)$ in 2 instead of C-9 $\left(\delta_{C} 82.6\right), C-11\left(\delta_{C} 73.5\right)$, and C-12 $\left(\delta_{\mathrm{C}} 76.1\right)$ in 4a-demethylpaspaline-3,4,4a-triol, indicating the replacement of two oxygenated carbons by a methylene (C-11) and an acetal carbon (C-9) in 2 . The HMBC correlations (Supplementary Material, Figure S16) from H-11, H-7, H-29, and H-28 to C-9 confirmed this deduction. The presence of an indole moiety, together with the HRESIMS data (Supplementary Material, Figure S19), indicated that 2 has a heptacyclic ring system. At this point, one more ring was required to fulfill the 11 double-bond equivalents, and an oxygen bridge was proposed according to the molecular formula. The oxygen bridge was assigned to connect C-9 and C-12 as deduced from distinctive deshielding of the C-12 $\left(\delta_{\mathrm{C}} 84.6\right)$ and C-9 $\left(\delta_{\mathrm{C}}\right.$ 109.8) in 2 compared to corresponding C-12 $\left(\delta_{C} 76.1\right)$ and $C-9\left(\delta_{C} 96.4-96.5\right)[25,26]$ with a free hydroxyl group. In the ROESY spectrum (Supplementary Material, Figure S18), correlations of $\mathrm{H}-16 / \mathrm{H}_{3}-26 / \mathrm{H}-5 \beta$, and $\mathrm{H}-$ $5 \alpha / \mathrm{H}_{3}-25 / \mathrm{H}-13 / \mathrm{H}-7 / \mathrm{H}-10 / \mathrm{H}_{3}-28$ determined the relative configuration of 2 , as shown in Figure 3 . In addition, the $\beta$ orientation of the oxygen bridge was also proved by the ROESY correlation of $\mathrm{H}-7 / \mathrm{H}_{2}-11$ with the aid of the 3D ball-and-stick molecular model. Thus, the planar structure of compound 2 was established and named penerpene L. The ECD curve (Figure 4 ) of compound $\mathbf{2}$ is similar to $\mathbf{1}$, indicating that the absolute configurations for the chiral carbons C-3, C-4, C-16, and C-13 in 1 were the same as those of 2 . The ECD calculation experiment also confirmed the above deduction (Figure 4), establishing the (3S,4S,7S,9S,10R,12R,13R,16S)-2 absolute configuration.

Compound 3 was assigned the molecular formula of $\mathrm{C}_{28} \mathrm{H}_{41} \mathrm{NO}_{2}$ by HRESIMS, indicating nine degrees of unsaturation. The ${ }^{1} \mathrm{H}$ NMR spectrum (Supplementary Material, Figure S21) displayed the typical pattern of a 3-substituted indole moiety with five aromatic protons 
at $\delta_{\mathrm{H}} 7.04(\mathrm{~s}, \mathrm{H}-2), 7.30(\mathrm{~d}, J=8.0, \mathrm{H}-7), 6.93(\mathrm{t}, J=7.6, \mathrm{H}-5), 7.02(\mathrm{t}, J=7.6, \mathrm{H}-6)$ and 7.55 $(\mathrm{d}, J=8.0, \mathrm{H}-4)$, as well as one clear olefinic proton at $\delta_{\mathrm{H}} 5.05(\mathrm{t}, \mathrm{H}-21)$, one oxygenated proton $\delta_{\mathrm{H}} 3.27(1 \mathrm{H}, \mathrm{H}-17)$, and five methyls at $\delta_{\mathrm{H}} 1.62(\mathrm{~s}, 24-\mathrm{Me}), 1.55(\mathrm{~s}, 23-\mathrm{Me}), 0.62(\mathrm{~s}$, 27-Me), 1.11 (s, 26-Me), 0.94 (s, 25-Me). The ${ }^{13} \mathrm{C}$ NMR data of 3 (Supplementary Materials, Figures S22 and S23) displayed 28 carbon signals, including ten aromatic or olefinic carbons (six protonated), five methyls, seven $\mathrm{sp}^{3}$ methylenes, three $\mathrm{sp}^{3}$ methines with one oxygenated, three non-protonated $\mathrm{sp}^{3}$ carbons with one oxygenated. These NMR spectra data indicated that 3 was very similar to emeniveol (16) [24] except that a methyl in $\mathrm{C}-26\left(\delta_{\mathrm{C} / \mathrm{H}} 16.3 / 1.11\right)$ and an oxygenated non-protonated sp ${ }^{3}$ carbon $\mathrm{C}-14\left(\delta_{\mathrm{C}} 76.4\right)$ in 3 replaced two olefinic carbons in emeniveol, suggesting that the exocyclic double bond in emeniveol changed to a methyl and an oxygenated non-protonated $\mathrm{sp}^{3}$ carbon. This obvious difference was supported by the HMBC correlations (Supplementary Material, Figure S25) from the $\mathrm{H}_{3}-26$ to $\mathrm{C}-14, \mathrm{C}-13\left(\delta_{\mathrm{C}} 42.0\right)$ and $\mathrm{C}-9\left(\delta_{\mathrm{C}} 42.9\right)$. The relative configuration of 3 was determined by the ROESY spectrum (Figure 3), and the correlations of 14-OH $/ \mathrm{H}_{3}-25 / \mathrm{H}_{3}-27 / 17-\mathrm{OH}$ and $\mathrm{H}-9 / \mathrm{H}_{3}-25$ suggested the same face of these protons, while the correlation of $\mathrm{H}-12 / \mathrm{H}-17$ indicated that these protons were on the opposite face to $17-\mathrm{OH}$. Thus, the planar structure of 3 was assigned, as shown in Figure 1. The absolute configuration of $\mathbf{3}$ was established as $(9 S, 12 S, 13 S, 14 S, 17 S, 18 S)-\mathbf{3}$ by comparison of its experimental ECD spectrum with the calculated ECD curves (Figure 4).

The molecular formula of compound 4 was established as $\mathrm{C}_{27} \mathrm{H}_{33} \mathrm{NO}_{4}$ on the basis of HRESIMS data, indicating 12 degrees of unsaturation. Analysis of the ${ }^{1} \mathrm{H}$ and ${ }^{13} \mathrm{C}$ NMR spectra (Tables 1 and 2, Supplementary Materials, Figures S30 and S31) of 4 suggested that its structure was closely related to that of penerpene $G$ [9], a previously reported indole diterpene with an unusual 6/5/5/6/6/7 hexacyclic ring system bearing a 1,3-dioxepane ring. The main difference between them was the replacement of an oxygenated nonprotonated sp $\mathrm{sp}^{3}$ carbon $\mathrm{C}-14\left(\delta_{\mathrm{C}} 78.3\right)$ in penerpene $\mathrm{G}$ by that of a sp ${ }^{3}$ methine $\left(\delta_{\mathrm{C}} 42.8\right)$ in 4. This assignment was confirmed by HMBC (Figure 2$)$ correlations from $\mathrm{H}-12\left(\delta_{\mathrm{H}} 5.54\right)$ and $\mathrm{H}-27\left(\delta_{\mathrm{H}} 0.82\right)$ to $\mathrm{C}-14$ and the COSY correction of $\mathrm{H}-14 / \mathrm{H}-15$. The ROESY corrections (Figure 3) of $\mathrm{H}_{3}-27 / \mathrm{H}-17 / \mathrm{H}-16 \beta$ and $\mathrm{H}-16 \alpha / \mathrm{H}_{3}-26 / \mathrm{H}-14 / \mathrm{H}-7 / \mathrm{H}-9$ assigned the same relative configuration of 4 as that of penerpene G. The ECD curves of compound 4 (Supplementary Material, Figure S43) are similar to that reported for penerpene G, containing strong positive CEs at a shorter wavelength (219 $\mathrm{nm}$ in 4 and $220 \mathrm{~nm}$ in penerpene G) and strong negative CEs at a longer wavelength ( $238 \mathrm{~nm}$ in 4 and $234 \mathrm{~nm}$ in penerpene $\mathrm{G}$ ), leading to the determination of the absolute configuration of 4 as $(3 S, 4 S, 7 S, 9 S, 14 R, 17 S)-4$.
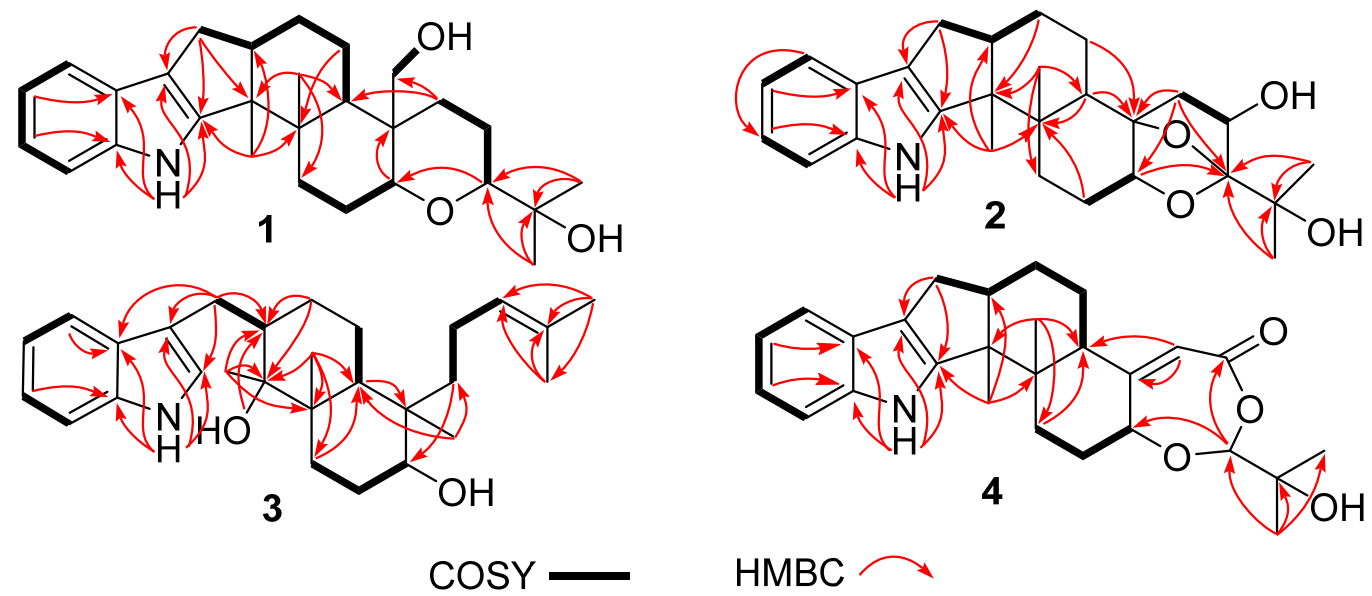

Figure 2. Key COSY and HMBC correlations of compounds 1-4. 

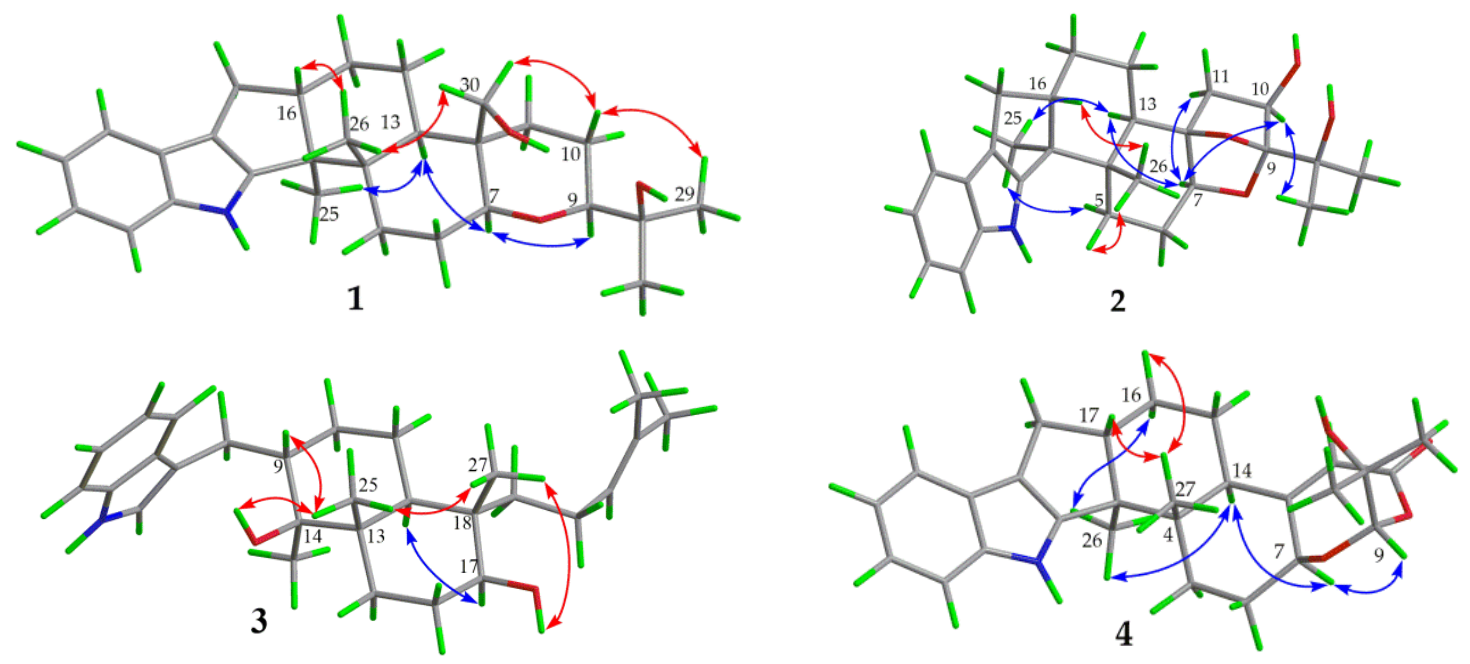

Figure 3. Key ROESY correlations of compounds 1-4.
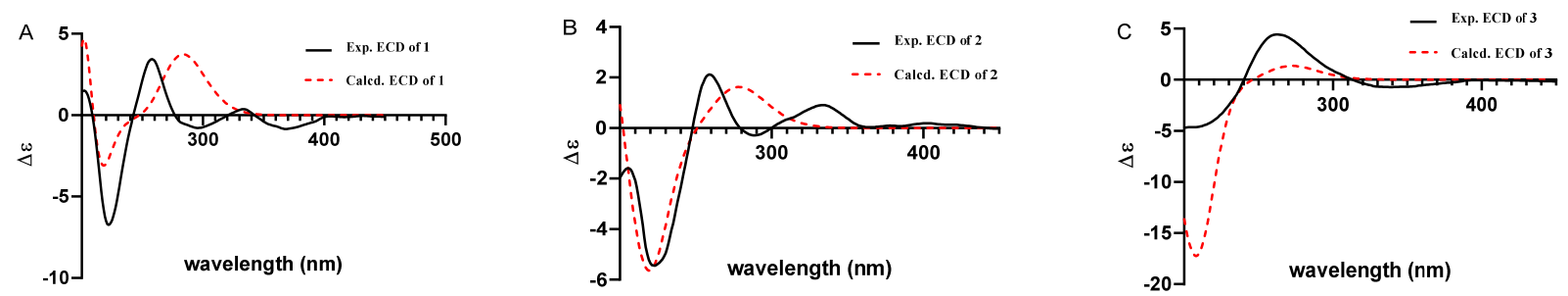

Figure 4. Experimental and calculated ECD curves for compound 1 (A); compound 2 (B); compound 3 (C).

Table 1. ${ }^{1} \mathrm{H}$ NMR (500 and $\left.600 \mathrm{MHz}\right)$ data of $\mathbf{1}-\mathbf{4}$ in DMSO- $d_{6}$.

\begin{tabular}{|c|c|c|c|c|}
\hline \multirow{2}{*}{ Position } & 1 & 2 & 3 & 4 \\
\hline & $\delta_{\mathrm{H}}(J$ in $\mathrm{Hz})$ & $\delta_{\mathrm{H}}(J$ in $\mathrm{Hz})$ & $\delta_{\mathrm{H}}(J$ in $\mathrm{Hz})$ & $\delta_{\mathrm{H}}(J$ in $\mathrm{Hz})$ \\
\hline 2 & & & $7.04(1 \mathrm{H}, \mathrm{s})$ & \\
\hline 4 & & & $7.55(1 \mathrm{H}, \mathrm{d}, 8.0)$ & \\
\hline \multirow[t]{2}{*}{5} & $1.92(1 \mathrm{H}, \mathrm{m})$ & $1.88(1 \mathrm{H}$, overlap $)$ & $6.93(1 \mathrm{H}, \mathrm{t}, 8.0)$ & $2.01(1 \mathrm{H}, \mathrm{m})$ \\
\hline & $1.80(1 \mathrm{H}, \mathrm{m})$ & $1.60(1 \mathrm{H}$, overlap $)$ & & $1.94(1 \mathrm{H}, \mathrm{m})$ \\
\hline \multirow[t]{2}{*}{6} & $1.51(1 \mathrm{H}, \mathrm{m})$ & $1.90(1 \mathrm{H}, \mathrm{m})$ & $7.02(1 \mathrm{H}, \mathrm{t}, 8.0)$ & $1.72(1 \mathrm{H}$, overlap $)$ \\
\hline & $1.62(1 \mathrm{H}$, overlap $)$ & $1.48(1 \mathrm{H}, \mathrm{m})$ & & $2.20(1 \mathrm{H}, \mathrm{m})$ \\
\hline 7 & $3.03(1 \mathrm{H}, \mathrm{dd}, 12.1,3.8)$ & $3.49(1 \mathrm{H}, \mathrm{dd}, 9.7,7.0)$ & $7.30(1 \mathrm{H}, \mathrm{d}, 8.0)$ & $4.46(1 \mathrm{H}, \mathrm{m})$ \\
\hline \multirow[t]{2}{*}{8} & & & $2.07(1 \mathrm{H}, \mathrm{m})$ & \\
\hline & & & $3.15(1 \mathrm{H}, \mathrm{d}, 13.3)$ & \\
\hline 9 & $3.10(1 \mathrm{H}, \mathrm{dd}, 12.0,2.7)$ & & $1.81(1 \mathrm{H}, \mathrm{m})$ & $5.05(1 \mathrm{H}, \mathrm{s})$ \\
\hline \multirow[t]{2}{*}{10} & $1.41(1 \mathrm{H}, \mathrm{m})$ & $4.04(1 \mathrm{H}, \mathrm{dd}, 2.1,6.7)$ & $1.10(1 \mathrm{H}, \mathrm{m})$ & \\
\hline & $1.62(1 \mathrm{H}$, overlap $)$ & & $1.65(1 \mathrm{H}, \mathrm{m})$ & \\
\hline \multirow[t]{2}{*}{11} & $1.74(1 \mathrm{H}, \mathrm{m})$ & $1.88(1 \mathrm{H}$, overlap $)$ & $1.26(2 \mathrm{H}, \mathrm{m})$ & \\
\hline & $1.69(1 \mathrm{H}, \mathrm{s})$ & $1.70(1 \mathrm{H}, \mathrm{m})$ & & \\
\hline 12 & & & $1.81(1 \mathrm{H}, \mathrm{m})$ & $5.54(1 \mathrm{H}, \mathrm{s})$ \\
\hline 13 & $1.39(1 \mathrm{H}, \mathrm{m})$ & $2.06(1 \mathrm{H}, \mathrm{dd}, 12.7,3.1)$ & & \\
\hline \multirow[t]{2}{*}{14} & $0.83(1 \mathrm{H}, \mathrm{m})$ & $1.74(1 \mathrm{H}, \mathrm{m})$ & & $2.55(1 \mathrm{H}, \mathrm{m})$ \\
\hline & $2.35(1 \mathrm{H}, \mathrm{m})$ & $1.64(1 \mathrm{H}, \mathrm{m})$ & & \\
\hline \multirow[t]{2}{*}{15} & $1.44(1 \mathrm{H}, \mathrm{m})$ & $1.78(1 \mathrm{H}, \mathrm{m})$ & $1.42(2 \mathrm{H}$, overlap $)$ & $1.53(1 \mathrm{H}, \mathrm{m})$ \\
\hline & $1.62(1 \mathrm{H}, \mathrm{m})$ & $1.60(1 \mathrm{H}$, overlap $)$ & & $1.62(1 \mathrm{H}$, overlap) \\
\hline \multirow[t]{2}{*}{16} & $2.61(1 \mathrm{H}, \mathrm{m})$ & $2.86(1 \mathrm{H}, \mathrm{m})$ & $1.24(1 \mathrm{H}$, overlap $)$ & $1.72(1 \mathrm{H}$, overlap $)$ \\
\hline & & & $1.54(1 \mathrm{H}, \mathrm{m})$ & $1.62(1 \mathrm{H}$, overlap $)$ \\
\hline
\end{tabular}


Table 1. Cont.

\begin{tabular}{|c|c|c|c|c|}
\hline \multirow{2}{*}{ Position } & 1 & 2 & 3 & 4 \\
\hline & $\delta_{\mathrm{H}}(J$ in $\mathrm{Hz})$ & $\delta_{\mathrm{H}}(J$ in $\mathrm{Hz})$ & $\delta_{\mathbf{H}}(J$ in $\mathbf{H z})$ & $\delta_{\mathrm{H}}(J$ in $\mathrm{Hz})$ \\
\hline \multirow[t]{2}{*}{17} & $2.54(1 \mathrm{H}, \mathrm{m})$ & $2.61(1 \mathrm{H}, \mathrm{m})$ & $3.27(1 \mathrm{H}, \mathrm{m})$ & $2.68(1 \mathrm{H}, \mathrm{m})$ \\
\hline & $2.21(1 \mathrm{H}, \mathrm{dd}, 12.3,10.9)$ & $2.29(1 \mathrm{H}, \mathrm{dd}, 11.2,12.6)$ & & \\
\hline \multirow[t]{2}{*}{18} & & & & $2.33(1 \mathrm{H}, \mathrm{m})$ \\
\hline & & & & $2.61(1 \mathrm{H}, \mathrm{m})$ \\
\hline \multirow[t]{2}{*}{19} & & & $1.02(1 \mathrm{H}, \mathrm{m})$ & \\
\hline & & & $1.47(1 \mathrm{H}, \mathrm{m})$ & \\
\hline 20 & $7.27(1 \mathrm{H}, \mathrm{d}, 7.5)$ & $7.27(1 \mathrm{H}, \mathrm{d}, 7.5)$ & $1.84(2 \mathrm{H}, \mathrm{m})$ & \\
\hline 21 & $6.91(1 \mathrm{H}, \mathrm{t}, 7.5)$ & $6.90(1 \mathrm{H}, \mathrm{m})$ & $5.05(1 \mathrm{H}, \mathrm{t}, 7.6)$ & $7.27(1 \mathrm{H}, \mathrm{m})$ \\
\hline 22 & $6.91(1 \mathrm{H}, \mathrm{t}, 7.5)$ & $6.94(1 \mathrm{H}, \mathrm{m})$ & & $6.92(1 \mathrm{H}, \mathrm{t}, 7.9)$ \\
\hline 23 & $7.24(1 \mathrm{H}, \mathrm{d}, 7.5)$ & $7.26(1 \mathrm{H}, \mathrm{d}, 7.5)$ & $1.55(3 \mathrm{H}, \mathrm{s})$ & $6.96(1 \mathrm{H}, \mathrm{t}, 7.9)$ \\
\hline 24 & & & $1.62(3 \mathrm{H}, \mathrm{s})$ & $7.27(1 \mathrm{H}, \mathrm{m})$ \\
\hline 25 & $0.94(3 \mathrm{H}, \mathrm{s})$ & $0.94(3 \mathrm{H}, \mathrm{s})$ & $0.94(3 \mathrm{H}, \mathrm{s})$ & \\
\hline 26 & $1.13(3 \mathrm{H}, \mathrm{s})$ & $1.17(3 \mathrm{H}, \mathrm{s})$ & $1.11(3 \mathrm{H}, \mathrm{s})$ & $1.01(3 \mathrm{H}, \mathrm{s})$ \\
\hline 27 & & & $0.62(3 \mathrm{H}, \mathrm{s})$ & $0.82(3 \mathrm{H}, \mathrm{s})$ \\
\hline 28 & $1.08(3 \mathrm{H}, \mathrm{s})$ & $1.26(3 \mathrm{H}, \mathrm{s})$ & & \\
\hline 29 & $1.03(3 \mathrm{H}, \mathrm{s})$ & $1.24(3 \mathrm{H}, \mathrm{s})$ & & $1.14(3 \mathrm{H}, \mathrm{s})$ \\
\hline \multirow[t]{2}{*}{30} & $3.69(1 \mathrm{H}, \mathrm{m})$ & & & $1.13(3 \mathrm{H}, \mathrm{s})$ \\
\hline & $3.86(1 \mathrm{H}, \mathrm{m})$ & & & \\
\hline $1-\mathrm{NH}$ & $10.55(1 \mathrm{H}, \mathrm{s})$ & $10.68(1 \mathrm{H}, \mathrm{s})$ & $10.67(1 \mathrm{H}, \mathrm{s})$ & $10.72(1 \mathrm{H}, \mathrm{s})$ \\
\hline $14-\mathrm{OH}$ & & & $4.04(1 \mathrm{H}, \mathrm{s})$ & \\
\hline $17-\mathrm{OH}$ & & & $4.22(1 \mathrm{H}, \mathrm{d}, 4.8)$ & \\
\hline $10-\mathrm{OH}$ & & $4.88(1 \mathrm{H}, \mathrm{s})$ & & \\
\hline $27-\mathrm{OH}$ & $4.16(1 \mathrm{H}, \mathrm{s})$ & & & \\
\hline $28-\mathrm{OH}$ & & & & $4.77(1 \mathrm{H}, \mathrm{s})$ \\
\hline $30-\mathrm{OH}$ & $4.07(1 \mathrm{H}, \mathrm{s})$ & & & \\
\hline
\end{tabular}

Compound 1 was measured with $500 \mathrm{MHz}{ }^{1} \mathrm{H}$ NMR. Compounds 2, 3, and 4 were measured with $600 \mathrm{MHz}{ }^{1} \mathrm{H}$ NMR.

Table 2. ${ }^{13} \mathrm{C}$ NMR (125 and $\left.150 \mathrm{MHz}\right)$ data of $\mathbf{1}-\mathbf{4}$ in DMSO- $d_{6}$.

\begin{tabular}{|c|c|c|c|c|}
\hline \multirow{2}{*}{ Position } & 1 & 2 & 3 & 4 \\
\hline & $\delta_{C}$ & $\delta_{C}$ & $\delta_{C}$ & $\delta_{C}$ \\
\hline 2 & $151.5, \mathrm{C}$ & $150.9, \mathrm{C}$ & $122.6, \mathrm{CH}$ & $150.2, \mathrm{C}$ \\
\hline 3 & $52.9, \mathrm{C}$ & $50.8, \mathrm{C}$ & $114.4, \mathrm{C}$ & $42.5, \mathrm{C}$ \\
\hline $3 a$ & & & 127.6, C & \\
\hline 4 & $39.9, \mathrm{C}$ & $38.5, \mathrm{C}$ & $118.7, \mathrm{CH}$ & $50.0, \mathrm{C}$ \\
\hline 5 & 32.7, $\mathrm{CH}_{2}$ & $30.6, \mathrm{CH}_{2}$ & $117.9, \mathrm{CH}$ & $30.8, \mathrm{CH}_{2}$ \\
\hline 6 & $24.5, \mathrm{CH}_{2}$ & $23.2, \mathrm{CH}_{2}$ & $120.6, \mathrm{CH}$ & $30.0, \mathrm{CH}_{2}$ \\
\hline 7 & $85.2, \mathrm{CH}$ & $78.2, \mathrm{CH}$ & $111.2, \mathrm{CH}$ & 83.0, $\mathrm{CH}$ \\
\hline $7 a$ & & & $136.3, \mathrm{C}$ & \\
\hline 8 & & & $25.5, \mathrm{CH}_{2}$ & \\
\hline 9 & $84.9, \mathrm{CH}$ & $109.8, \mathrm{C}$ & $42.9, \mathrm{CH}$ & 102.3, CH \\
\hline 10 & 25.7, $\mathrm{CH}_{2}$ & 73.1, CH & 27.2, $\mathrm{CH}_{2}$ & \\
\hline 11 & $23.8, \mathrm{CH}_{2}$ & 43.7, $\mathrm{CH}_{2}$ & $20.7, \mathrm{CH}_{2}$ & $166.8, \mathrm{C}$ \\
\hline 12 & $48.8, \mathrm{C}$ & $84.6, \mathrm{C}$ & $41.1, \mathrm{CH}$ & $113.1, \mathrm{CH}$ \\
\hline 13 & $46.9, \mathrm{CH}$ & $38.8, \mathrm{CH}$ & $42.0, \mathrm{C}$ & 163.1, C \\
\hline 14 & $31.8, \mathrm{CH}_{2}$ & $26.2, \mathrm{CH}_{2}$ & $76.4, \mathrm{C}$ & $42.8, \mathrm{CH}$ \\
\hline 15 & $21.3, \mathrm{CH}_{2}$ & $25.2, \mathrm{CH}_{2}$ & $29.9, \mathrm{CH}_{2}$ & $25.6, \mathrm{CH}_{2}$ \\
\hline 16 & $49.0, \mathrm{CH}$ & $49.0, \mathrm{CH}$ & $28.5, \mathrm{CH}_{2}$ & $23.9, \mathrm{CH}_{2}$ \\
\hline 17 & 27.3, $\mathrm{CH}_{2}$ & $27.1, \mathrm{CH}_{2}$ & $71.5, \mathrm{CH}$ & $48.7, \mathrm{CH}$ \\
\hline 18 & 116.1, C & $115.9, \mathrm{C}$ & $40.6, \mathrm{C}$ & $26.9, \mathrm{CH}_{2}$ \\
\hline 19 & $124.6, \mathrm{C}$ & $124.4, \mathrm{C}$ & 37.0, $\mathrm{CH}_{2}$ & $115.8, \mathrm{C}$ \\
\hline 20 & $117.8 \mathrm{CH}$ & 117.7, CH & $21.2, \mathrm{CH}_{2}$ & $124.3, \mathrm{C}$ \\
\hline 21 & 118.6, $\mathrm{CH}$ & $118.5, \mathrm{CH}$ & 125.3, $\mathrm{CH}$ & 117.7, $\mathrm{CH}$ \\
\hline
\end{tabular}


Table 2. Cont.

\begin{tabular}{ccccc}
\hline \multirow{2}{*}{ Position } & $\mathbf{1}$ & $\mathbf{2}$ & $\mathbf{3}$ & $\mathbf{4}$ \\
\cline { 2 - 5 } & \multicolumn{1}{c}{$\boldsymbol{\delta}_{\mathrm{C}}$} & $\delta_{\mathrm{C}}$ & $\delta_{\mathrm{C}}$ & $\delta_{\mathrm{C}}$ \\
\cline { 2 - 5 } 22 & $119.3, \mathrm{CH}$ & $119.4, \mathrm{CH}$ & $129.8, \mathrm{C}$ & $118.5, \mathrm{CH}$ \\
23 & $112.0, \mathrm{CH}$ & $111.9, \mathrm{CH}$ & $17.5, \mathrm{CH}_{3}$ & $119.5, \mathrm{CH}$ \\
24 & $140.4, \mathrm{C}$ & $140.1, \mathrm{C}$ & $25.6, \mathrm{CH}_{3}$ & $111.9, \mathrm{CH}$ \\
25 & $14.8, \mathrm{CH}_{3}$ & $14.4, \mathrm{CH}_{3}$ & $14.6, \mathrm{CH}_{3}$ & $140.1, \mathrm{C}$ \\
26 & $19.5, \mathrm{CH}_{3}$ & $16.7, \mathrm{CH}_{3}$ & $16.3, \mathrm{CH}_{3}$ & $14.7, \mathrm{CH}_{3}$ \\
27 & $70.7, \mathrm{C}$ & $70.9, \mathrm{C}$ & $17.2, \mathrm{CH}_{3}$ & $15.4, \mathrm{CH}_{3}$ \\
28 & $26.9, \mathrm{CH}_{3}$ & $26.4, \mathrm{CH}_{3}$ & & $69.9, \mathrm{C}$ \\
29 & $24.9, \mathrm{CH}_{3}$ & $24.7, \mathrm{CH}_{3}$ & & $24.0, \mathrm{CH}_{3}$ \\
30 & $58.5, \mathrm{CH}_{2}$ & & & $23.8, \mathrm{CH}_{3}$ \\
\hline
\end{tabular}

Compound 1 was measured with $125 \mathrm{MHz}{ }^{13} \mathrm{C}$ NMR. Compounds 2, 3, and 4 were measured with $150 \mathrm{MHz}$ ${ }^{13} \mathrm{C}$ NMR.

\subsection{Biological Assay}

The cytotoxic activities of compounds 1-16 were conducted by the MTT assay method [27] using cisplatin as the positive control. All compounds were tested against the human cervical cancer cell line HeLa, human gastric cancer cell line SGC-7901, human lung carcinoma cell line A549, and human liver cancer cell line BeL-7402. The results (Table 3) indicated that compound 9 exhibited the most pronounced activity against BeL-7402 with an $\mathrm{IC}_{50}$ value of $5.3 \mu \mathrm{M}$ and was comparable to that of positive control cisplatin $\left(\mathrm{IC}_{50} 4.1 \mu \mathrm{M}\right)$. Compound 9 also displayed moderate cytotoxic activity against A549. While compounds 4 showed low cytotoxicity against HeLa. Compound $\mathbf{1 5}$ displayed mild inhibitory activity against HeLa, A549, and BeL-7402 $\left(\mathrm{IC}_{50}=24.4-40.6 \mu \mathrm{M}\right)$. The remaining compounds were found to be inactive against the three cell lines. All the tested compounds 1-16 were inactive against the cell line SGC-7901. The loss of a hydroxyl at C-14 suggested being a determinant of cytotoxicity shown by compound 4 against HeLa (4 vs. penerpene $G$ [9]). It is worth mentioning that it was the first-time report of the cytotoxicity of epipaxilline (9) [18]. Arintari et al. [19] and Sallam et al. [28] demonstrated the cytotoxicities of emindole SB (15) against human breast cell line MCF-7, murine lymphoma cell line L5178Y, and the human embryonic kidney cell line HEK-293, which provides emindole SB (15) a meaningful pharmacophore for further biological studies.

Table 3. Cytotoxicity of compounds 4, 9, and 15.

\begin{tabular}{cccc}
\hline \multirow{2}{*}{ Compound } & & IC $_{\mathbf{5 0}}(\boldsymbol{\mu M})$ & \\
\cline { 2 - 4 } & HeLa & A549 & BeL-7402 \\
\hline $\mathbf{4}$ & 36.3 & $>50$ & $>50$ \\
$\mathbf{9}$ & $>50$ & 28.4 & 5.3 \\
$\mathbf{1 5}$ & 33.1 & 24.4 & 40.6 \\
Cisplatin $^{\text {a }}$ & 8.6 & 4.5 & 4.1 \\
\hline a Positive control & & &
\end{tabular}

a Positive control.

Compounds 1-16 were also tested for their antibacterial activity against Escherichia coli ATCC 25922, Staphylococcus aureus ATCC 6538, Listeria monocytogenes ATCC 1911, and Bacillus subtilis ATCC 6633 using the 96-well microtiter plates method [29] reported previously and using ampicillin as a positive control. The results (Table 4) revealed that six compounds $5,7,10,12,14$, and 15 showed moderate inhibitory activity against $S$. aureus ATCC 6538. Emindole SB (15) displayed reported selectivity toward S. aureus ATCC 33591 $(\mathrm{MIC}=6.25 \mu \mathrm{g} / \mathrm{mL})$ [21]. Compound 7 showed reasonable antibacterial activity against B. subtilis ATCC $6633(\mathrm{MIC}=16 \mu \mathrm{g} / \mathrm{mL})$, but compounds 5, 10, and 12 exhibited lower inhibitory. The results of the rest ten compounds $(\mathbf{1 - 4}, \mathbf{6}, \mathbf{8 - 9}, \mathbf{1 1}, \mathbf{1 3}$, and 16) did not show remarkable antibacterial activities (MIC $>128 \mu \mathrm{g} / \mathrm{mL}$ ) against $S$. aureus and B. subtilis. In this assay, none of these compounds showed inhibitory activity against E. coli ATCC 25922 and L. monocytogenes ATCC 1911 (MIC > $128 \mu \mathrm{g} / \mathrm{mL}$ ). 
Table 4. Antibacterial activities of compounds 5, 7, 10, 12, 14, and 15.

\begin{tabular}{ccc}
\hline \multirow{2}{*}{ Compound } & \multicolumn{2}{c}{ MIC $(\mu \mathrm{g} / \mathrm{mL})$} \\
\cline { 2 - 3 } & $\begin{array}{c}\text { Staphylococcus aureus } \\
\text { ATCC } \mathbf{6 5 3 8}\end{array}$ & $\begin{array}{c}\text { Bacillus subtilis } \\
\text { ATCC } \mathbf{6 6 3 3}\end{array}$ \\
\hline $\mathbf{5}$ & 128 & 32 \\
$\mathbf{7}$ & 64 & 16 \\
$\mathbf{1 0}$ & 64 & 64 \\
$\mathbf{1 2}$ & 64 & 128 \\
$\mathbf{1 5}$ & 64 & $>128$ \\
Ampicillin ${ }^{\text {a }}$ & 32 & $>128$ \\
& $<1$ & $<1$ \\
\hline a Positive control. & &
\end{tabular}

${ }^{a}$ Positive control.

\section{Experimental}

\subsection{General Experimental Procedures}

NMR spectra were recorded on Bruker AV-500 and Bruker AV-600 spectrometers (Bruker, Bremen, Germany) with TMS as an internal standard. The mass spectrometric (HRESIMS) data were acquired using an API QSTAR Pulsar mass spectrometer (Bruker, Bremen, Germany) and an AB SCIEX Trip TOF 5600+ mass spectrometer (SCIEX, Framingham, MA, USA). Optical rotations were measured with a JASCO P-1020 digital polarimeter (Jasco, Tokyo, Japan). IR spectra were recorded on a Shimadzu UV2550 spectrophotometer (Shimadzu, Kyoto, Japan). UV spectra and ECD data were collected using a JASCO J-715 spectropolarimeter (Jasco, Tokyo, Japan). Semipreparative HPLC was carried out using an ODS column (YMC-pack ODS-A, $10 \times 250$ mm, 5 m, 4 mL/min, YMC, Kyoto, Japan).

\subsection{Fungus Material}

The fungus Penicillium sp. KFD28 (GenBank accession No. MK934323) was isolated from a bivalve mollusk, Meretrix lusoria, collected from Haikou Bay, Hainan province, in China. A reference culture of Penicillium sp. KFD28 is deposited in our laboratory and maintained at $-80^{\circ} \mathrm{C}$.

\subsection{Culture Conditions}

The fungus Penicillium sp. KFD28 was cultured in $200 \times 1000 \mathrm{~mL}$ Erlenmeyer flasks containing $100 \mathrm{~g}$ rice and $100 \mathrm{~mL}$ of water $(33 \mathrm{~g}$ sea salt, $5 \mathrm{~g}$ L-tryptophan per liter pure water). The fungus was cultured in the medium and incubated at room temperature for thirty days.

\subsection{Extraction and Isolation}

The fermented material was extracted three times with EtOAc to obtain $300 \mathrm{~g}$ crude extract. The extract was extracted between petroleum ether and $90 \%$ methanol (1:1) to remove the oil. The secondary metabolites extract $(46 \mathrm{~g})$ was subjected to a silica gel VLC column, eluting with a stepwise gradient of petroleum ether-EtOAc $(10: 1,8: 1,6: 1,4: 1,2: 1$, 1:1, 1:2, v/v) to yield ten subfractions (Fr. 1-Fr. 10).

Fr. $3(4.2 \mathrm{~g})$ was applied to ODS silica gel with gradient elution of $\mathrm{MeOH}-\mathrm{H}_{2} \mathrm{O}$ $(1: 4,3: 7,2: 3,1: 1,3: 2,7: 3,4: 1,9: 1,0: 1, v / v)$ and afforded nine subfractions (Fr. 3-1-Fr. 39). Fr. 3-9 (182 mg) was applied to semipreparative HPLC (YMC-pack ODS-A, $5 \mu \mathrm{m}$; $\left.10 \times 250 \mathrm{~mm} ; 85 \% \mathrm{MeOH} / \mathrm{H}_{2} \mathrm{O} ; 4 \mathrm{~mL} / \mathrm{min}\right)$ to obtain compounds $16\left(t_{\mathrm{R}} 20.0 \mathrm{~min}, 1.0 \mathrm{mg}\right)$, $11\left(t_{\mathrm{R}} 23.0 \mathrm{~min}, 9.7 \mathrm{mg}\right)$, and $8\left(t_{\mathrm{R}} 32.0 \mathrm{~min}, 20.8 \mathrm{mg}\right)$. Fr. $5(3.8 \mathrm{~g})$ was applied to ODS silica gel with gradient elution of $\mathrm{MeOH}-\mathrm{H}_{2} \mathrm{O}(1: 9,1: 4,3: 7,2: 3,1: 1,3: 2,7: 3,4: 1,9: 1,0: 1, v / v)$ to get ten subfractions. Fr. 5-10 (323 mg) was purified by semipreparative HPLC (YMCpack ODS-A, $\left.5 \mu \mathrm{m} ; 10 \times 250 \mathrm{~mm} ; 80 \% \mathrm{MeOH} / \mathrm{H}_{2} \mathrm{O} ; 4 \mathrm{~mL} / \mathrm{min}\right)$ to give compound $15\left(t_{\mathrm{R}}\right.$ $28.5 \mathrm{~min}, 53.4 \mathrm{mg})$. Fr. $6(5.1 \mathrm{~g})$ was separated by ODS silica gel with gradient elution of $\mathrm{MeOH}-\mathrm{H}_{2} \mathrm{O}(1: 9,1: 4,3: 7,2: 3,1: 1,3: 2,7: 3,4: 1,9: 1,0: 1, v / v)$ to yield ten subfractions (Fr. 6-1Fr. 6-10). Fr. 6-10 (1.1 g) was subjected to semipreparative HPLC (YMC-pack ODS-A, $5 \mu \mathrm{m}$; 
$10 \times 250 \mathrm{~mm} ; 65 \% \mathrm{MeCN} / \mathrm{H}_{2} \mathrm{O} ; 4 \mathrm{~mL} / \mathrm{min}$ ) to give compounds 5 ( $t_{\mathrm{R}} 11.5 \mathrm{~min}, 182.6 \mathrm{mg}$ ) and $6\left(t_{\mathrm{R}} 18.0 \mathrm{~min}, 25.8 \mathrm{mg}\right)$. Fr. $7(4.7 \mathrm{~g})$ was applied to ODS silica gel with gradient elution of $\mathrm{MeOH}-\mathrm{H}_{2} \mathrm{O}(1: 9,1: 4,3: 7,2: 3,1: 1,3: 2,7: 3,4: 1,9: 1,0: 1, v / v)$, ten subfractions (Fr. 7-1-7-10) were obtained. Fr. 7-7 (587 mg) was eluted with $\mathrm{MeCN}-\mathrm{H}_{2} \mathrm{O}$ through ODS silica gel $(3: 7,2: 3,1: 1,3: 2,7: 3, v / v)$ to afford five subfractions (Fr. 7-7-1-7-7-5). Fr. 7-7-4 (117 mg) was separated by semipreparative HPLC (YMC-pack ODS-A, $5 \mu \mathrm{m} ; 10 \times 250 \mathrm{~mm} ; 70 \%$ $\mathrm{MeCN} / \mathrm{H}_{2} \mathrm{O} ; 4 \mathrm{~mL} / \mathrm{min}$ ) to give compounds $2\left(t_{\mathrm{R}} 8.6 \mathrm{~min}, 0.6 \mathrm{mg}\right), 3\left(t_{\mathrm{R}} 9.4 \mathrm{~min}, 1.8 \mathrm{mg}\right), 1$ $\left(t_{\mathrm{R}} 10.4 \mathrm{~min}, 2.0 \mathrm{mg}\right), \mathbf{1 3}\left(t_{\mathrm{R}} 11.6 \mathrm{~min}, 0.6 \mathrm{mg}\right), \mathbf{1 4}\left(t_{\mathrm{R}} 13.5 \mathrm{~min}, 9.9 \mathrm{mg}\right)$, and $\mathbf{7}\left(t_{\mathrm{R}} 22.0 \mathrm{~min}\right.$, $8.1 \mathrm{mg}$ ). Fr. 7-7-5 (137 mg) was applied to semipreparative HPLC (YMC-pack ODS-A, $5 \mu \mathrm{m}$; $\left.10 \times 250 \mathrm{~mm} ; 70 \% \mathrm{MeOH} / \mathrm{H}_{2} \mathrm{O} ; 4 \mathrm{~mL} / \mathrm{min}\right)$ to get compounds $9\left(t_{\mathrm{R}} 14.0 \mathrm{~min}, 2.0 \mathrm{mg}\right)$ and $10\left(t_{\mathrm{R}} 18.4 \mathrm{~min}, 3.4 \mathrm{mg}\right)$. Fr. $8(5.8 \mathrm{~g})$ was subjected to ODS silica gel with gradient elution of $\mathrm{MeOH}-\mathrm{H}_{2} \mathrm{O}(1: 4,3: 7,2: 3,1: 1,3: 2,7: 3,4: 1,9: 1,0: 1, v / v)$ to get nine subfractions. Then, Fr. 8-7 (925 mg) was eluted through ODS silica gel column of $\mathrm{MeCN}_{-} \mathrm{H}_{2} \mathrm{O}(1: 4,3: 7,2: 3$, $1: 1,3: 2, v / v)$ to get five subfractions (Fr. 8-7-1-8-7-5). Fr. 8-7-5 (29 mg) was separated by a semipreparative HPLC (YMC-pack ODS-A, $5 \mu \mathrm{m} ; 10 \times 250 \mathrm{~mm} ; 60 \% \mathrm{MeCN} / \mathrm{H}_{2} \mathrm{O}$; $4 \mathrm{~mL} / \mathrm{min})$ to yield compound $4\left(t_{\mathrm{R}} 13.0 \mathrm{~min}, 2.2 \mathrm{mg}\right)$. Fr. $8-9(812 \mathrm{mg})$ was eluted by a semipreparative HPLC (YMC-pack ODS-A, $5 \mu \mathrm{m} ; 10 \times 250 \mathrm{~mm} ; 80 \% \mathrm{MeOH} / \mathrm{H}_{2} \mathrm{O}$; $4 \mathrm{~mL} / \mathrm{min})$ to afford compound $12\left(t_{\mathrm{R}} 30.0 \mathrm{~min}, 157.9 \mathrm{mg}\right)$.

Penerpene K (1): Yellow oil; $[\alpha]_{\mathrm{D}}^{25}-23.0(c 0.01, \mathrm{MeOH}) ; \mathrm{UV}(\mathrm{MeOH}) \lambda_{\max }(\log \varepsilon): 231$ (3.41), 280 (2.77) nm; ECD (0.38 mM, MeOH) $\lambda_{\max }(\Delta \varepsilon)$ : 204 (2.46), 223 (-6.08), 255 (2.89); $294(-0.62) \mathrm{nm}$; IR (KBr) $v_{\max }: 3412,2933,1640,1606,1458,1382,1307,1257,1212,1160$, and $1088 \mathrm{~cm}^{-1} ;{ }^{1} \mathrm{H}$ and ${ }^{13} \mathrm{C}$ NMR spectral data, Tables 1 and 2; HRESIMS $\mathrm{m} / z 476.2565$ $\left([\mathrm{M}+\mathrm{K}]^{+}\right.$calcd 476.2562$)$.

Penerpene L (2): Yellow oil; $[\alpha]_{\mathrm{D}}^{25}-22.0(c 0.01, \mathrm{MeOH}) ; \mathrm{UV}(\mathrm{MeOH}) \lambda_{\max }(\log \varepsilon): 231$ (3.15), $282(2.41) \mathrm{nm}$; ECD (1.10 mM, MeOH) $\lambda_{\max }(\Delta \varepsilon): 203$ (0.14), $224(-1.63), 260(0.58)$, $292(-0.08), 330(0.24) \mathrm{nm}$; IR (KBr) $v_{\max }: 3411,2935,1645,1604,1452,1381,1307,1256$, 1158,1074 , and $1018 \mathrm{~cm}^{-1} ;{ }^{1} \mathrm{H}$ and ${ }^{13} \mathrm{C}$ NMR spectral data, Tables 1 and 2; HRESIMS $\mathrm{m} / \mathrm{z}$ 476.2210 $\left([\mathrm{M}+\mathrm{K}]^{+}\right.$calcd 476.2198).

Penerpene M (3): White powder; $[\alpha]_{\mathrm{D}}^{25}+15.0\left(\right.$ c 0.01, MeOH); UV (MeOH) $\lambda_{\max }(\log \varepsilon)$ : 226 (2.84), 261 (2.32) nm; ECD (1.15 mM, MeOH) $\lambda_{\max }(\Delta \varepsilon): 198$ (1.32), $202(-1.27), 221$ (-0.92), 263 (0.94) nm; IR (KBr) $v_{\max }: 3383,2931,1648,1609,1575,1357,1316,1257,1208$, 1160, 1118, and $1044 \mathrm{~cm}^{-1} ;{ }^{1} \mathrm{H}$ and ${ }^{13} \mathrm{C}$ NMR spectral data, Tables 1 and 2; HRESIMS m/z 446.3020 ([M+Na $]^{+}$calcd 446.3030).

Penerpene N (4): Yellow oil; $[\alpha]_{\mathrm{D}}^{25}+21.0(c 0.01, \mathrm{MeOH}) ; \mathrm{UV}(\mathrm{MeOH}) \lambda_{\max }(\log \varepsilon): 231$ (3.05), $277(2.44) \mathrm{nm} ; \mathrm{ECD}(0.77 \mathrm{mM}, \mathrm{MeOH}) \lambda_{\max }(\Delta \varepsilon): 197$ (-3.23), 219 (4.27), 238 (-6.39), 257 (3.21) nm; IR (KBr) $v_{\max }: 3415,2937,1641,1408,1110,1408,1110,1042,922,852,688$, and $565 \mathrm{~cm}^{-1} ;{ }^{1} \mathrm{H}$ and ${ }^{13} \mathrm{C}$ NMR spectral data, Tables 1 and 2; HRESIMS $\mathrm{m} / z 434.2338$ ([M-H] $]^{-}$calcd 434.2331).

\section{Conclusions}

To summarize, based on the OSMAC culture strategy, four new indole-diterpenoids were isolated from the marine-derived fungus Penicillium sp. KFD28 secondary metabolites. The absolute configurations of new compounds 1-3 were determined by spectroscopic methods coupled with experimental and calculated ECD. New compound 4 showed mild cytotoxicity against the human cervical cancer cell line HeLa. Notably, compound 9 exhibited strong cytotoxic activity against the human liver cancer cell line BeL-7402 with $\mathrm{IC}_{50}$ values of $5.3 \mu \mathrm{M}$, indicating that compound 9 deserves further study for its therapeutic potential to develop new anti-hepatoma drugs. Compound 7 showed pronounced antibacterial activity against Bacillus subtilis with MIC values of $16 \mu \mathrm{g} / \mathrm{mL}$, which had the potential to become an antibiotic. However, the mechanisms causing cytotoxicity and bacteria restraint were unknown and require further study. In general, this study expanded the application of the OSMAC method to increase the chemical and biological diversity of natural products isolated from the Penicillium sp. KFD28. 
Supplementary Materials: The following are available online at https:/ / www.mdpi.com/article/10 .3390/md19110613/s1, Figures S1-S38: 1D NMR, 2D NMR, IR, and HRESIMS spectra of the new compounds 1-4; Figure S39: the picture of strain Penicillium sp. KFD28; Figure S40: Separation flow chart of fungus Penicillium sp. KFD28 secondary metabolites extract; Figure S41: HPLC chromatograms of the EtOAc extracts monitored at wavelengths of $230 \mathrm{~nm}$ and $274 \mathrm{~nm}$; Figure S42: HPLC chromatograms of the EtOAc extracts using Rice solid medium with L-tryptophan, and compounds 6, 12, 14, 8, and 11 monitored at wavelengths of $230 \mathrm{~nm}$ and $274 \mathrm{~nm}$; Figure S43: ECD curve for compound 4 and computation section of compounds $1-3$.

Author Contributions: L.-T.D. contributed to fungal fermentation, compounds purification, and structural elucidation. L.Y. and F.-D.K. were responsible for chemical computation and preparation of the paper. Q.-Y.M. and Q.-Y.X. contributed to the bioassays and identified the fungal strain. H.-F.D. revised the paper. Z.-F.Y. and Y.-X.Z. designed the work and revised the paper. All authors have read and agreed to the published version of the manuscript.

Funding: This research was supported by the Natural Science Foundation of Hainan Province (2019CXTD411), Financial Fund of the Ministry of Agriculture and Rural Affairs, P.R. of China (NFZX2021), China Agriculture Research System of MOF and MARA (CARS-21), Central Publicinterest Scientific Institution Basal Research Fund for Chinese Academy of Tropical Agricultural Sciences (1630052017002, 1630052021019).

Institutional Review Board Statement: Not applicable.

Conflicts of Interest: The authors declare no conflict of interest.

\section{References}

1. Lindequist, U. Marine-derived pharmaceuticals-challenges and opportunities. Biomol. Ther. 2016, 24, 561-571. [CrossRef] [PubMed]

2. Carroll, A.R.; Copp, B.R.; Davis, R.A.; Keyzers, R.A.; Prinsep, M.R. Marine natural products. Nat. Prod. Rep. 2019, 36, 122-173. [CrossRef]

3. Hu, Y.W.; Chen, J.H.; Hu, G.P.; Yu, J.C.; Zhu, X.; Lin, Y.C.; Chen, S.P.; Yuan, J. Statistical research on the bioactivity of new marine natural products discovered during the 28 years from 1985 to 2012. Mar. Drugs 2015, 13, 202-221. [CrossRef]

4. Li, T.T.; Wang, Y.; Li, L.; Tang, M.Y.; Meng, Q.H.; Zhang, C.; Hua, E.B.; Pei, Y.H.; Sun, Y. New cytotoxic cytochalasans from a plant-associated fungus Chaetomium globosum kz-19. Mar. Drugs 2021, 19, 438. [CrossRef]

5. Kubota, T.; Nakamura, K.; Kurimoto, S.I.; Sakai, K.; Fromont, J.; Gonoi, T.; Kobayashi, J. Zamamidine D, a manzamine alkaloid from an okinawan Amphimedon sp. marine sponge. J. Nat. Prod. 2017, 80, 1196-1199. [CrossRef]

6. Kong, F.D.; Zhang, S.L.; Zhou, S.Q.; Ma, Q.Y.; Xie, Q.Y.; Chen, J.P.; Li, J.H.; Zhou, L.M.; Yuan, J.Z.; Hu, Z.; et al. Quinazoline-containing indole alkaloids from the marine-derived fungus Aspergillus sp. HNMF114. J. Nat. Prod. 2019, 82, 3456-3463. [CrossRef]

7. Guo, Y.W.; Liu, X.J.; Yuan, J.; Li, H.J.; Mahmud, T.; Hong, M.T.; Yu, J.C.; Lan, W.J. L-tryptophan induces a marine-derived Fusarium sp. to produce indole alkaloids with activity against the Zika virus. J. Nat. Prod. 2020, 83, 3372-3380. [CrossRef] [PubMed]

8. Kong, F.D.; Fan, P.; Zhou, L.M.; Ma, Q.Y.; Xie, Q.Y.; Zheng, H.Z.; Zhang, R.S.; Yuan, J.Z.; Dai, H.F.; Luo, D.Q.; et al. Penerpenes A-D, four indole terpenoids with potent protein tyrosine phosphatase inhibitory activity from the marine-derived fungus Penicillium sp. KFD28. Org. Lett. 2019, 21, 4864-4867. [CrossRef]

9. Zhou, L.M.; Kong, F.D.; Fan, P.; Ma, Q.Y.; Xie, Q.Y.; Li, J.H.; Zheng, H.Z.; Zheng, Z.H.; Yuan, J.Z.; Dai, H.F.; et al. Indolediterpenoids with protein tyrosine phosphatase inhibitory activities from the marine-derived fungus Penicillium sp. KFD28. J. Nat. Prod. 2019, 82, 2638-2644. [CrossRef] [PubMed]

10. Bode, H.B.; Bethe, B.; Höfs, R.; Zeeck, A. Big effects from small changes: Possible ways to explore nature's chemical diversity. ChemBioChem 2002, 3, 619-627. [CrossRef]

11. Xu, W.; Gavia, D.J.; Tang, Y. Biosynthesis of fungal indole alkaloids. Nat. Prod. Rep. 2014, 31, 1474-1487. [CrossRef] [PubMed]

12. Huang, L.H.; Xu, M.Y.; Li, H.J.; Li, J.Q.; Chen, Y.X.; Ma, W.Z.; Li, Y.P.; Xu, J.; Yang, D.P.; Lan, W.J. Amino acid-directed strategy for inducing the marine-derived fungus Scedosporium apiospermum F41-1 to maximize alkaloid diversity. Org. Lett. 2017, 19, 4888-4891. [CrossRef] [PubMed]

13. Liu, S.S.; Yang, L.; Kong, F.D.; Zhao, J.H.; Yao, L.; Yuchi, Z.G.; Ma, Q.Y.; Xie, Q.Y.; Zhou, L.M.; Guo, M.F.; et al. Three new quinazoline-containing indole alkaloids from the marine-derived fungus Aspergillus sp. HNMF114. Front. Microbiol. 2021, 12. [CrossRef]

14. Hosoe, T.; Nozawa, K.; Udagawa, S.I.; Nakajima, S.; Kawai, K.I. Structures of new indoloditerpenes, possible biosynthetic precursors of the tremorgenic mycotoxins, penitrems, from Penicillium crustosum. Chem. Phar. Bull. 2008, 38, 3473-3475. [CrossRef]

15. Nozawa, K.; Horie, Y.; Udagawa, S.I.; Kawai, K.I.; Yamazaki, M. Isolation of a new tremorgenic indologiterpene, $1^{\prime}$ O-Acetylpaxilline, from Emericella striata and distribution of paxilline in Emericella spp. Chem. Phar. Bull. 1989, 37, 1387-1389. [CrossRef] 
16. Belofsky, G.N.; Gloer, J.B.; Wicklow, D.T.; Dowd, P.F. Antiinsectan alkaloids: Shearinines A-C and a new paxilline derivative from the Ascostromata of Eupenicillium Shearii. Tetrahedron 1995, 51, 3959-3968. [CrossRef]

17. Fan, Y.Q.; Wang, Y.; Liu, P.P.; Fu, P.; Zhu, T.H.; Wang, W.; Zhu, W.M. Indole-diterpenoids with anti-H1N1 activity from the aciduric fungus Penicillium camemberti OUCMDZ-1492. J. Nat. Prod. 2013, 76, 1328-1336. [CrossRef]

18. Chen, M.Y.; Xie, Q.Y.; Kong, F.D.; Ma, Q.Y.; Zhou, L.M.; Yuan, J.Z.; Dai, H.F.; Wu, Y.G.; Zhao, Y.X. Two new indole-diterpenoids from the marine-derived fungus Penicillium sp. KFD28. J. Asian Nat. Prod. Res. 2020, 1-8. [CrossRef]

19. Ariantari, N.P.; Ancheeva, E.; Wang, C.Y.; Mándi, A.; Knedel, T.O.; Kurtán, T.; Chaidir, C.; Müller, W.E.G.; Kassack, M.U.; Janiak, C.; et al. Indole diterpenoids from an endophytic Penicillium sp. J. Nat. Prod. 2019, 82, 1412-1423. [CrossRef]

20. Munday-Finch, S.C.; Wilkins, A.L.; Miles, C.O. Isolation of paspaline B, an indole-diterpenoid from Penicillium paxilli. Phytochemistry 1996, 41, 327-332. [CrossRef]

21. Liang, Z.Y.; Shen, N.X.; Zhou, X.J.; Zheng, Y.Y.; Chen, M.; Wang, C.Y. Bioactive indole diterpenoids and polyketides from the marine-derived fungus penicillium javanicum. Chem. Nat. Comp. 2020, 56, 379-382. [CrossRef]

22. Yamaguchi, T.; Nozawa, K.; Hosoe, T.; Nakajima, S.; Kawai, K.I. Indoloditerpenes related to tremorgenic mycotoxins, penitrems, from Penicillium crustosum. Phytochemistry 1993, 32, 1177-1181. [CrossRef]

23. Nozawa, K.; Nakajima, S.; Kawai, K.I.; Udagawa, S.I. Isolation and structures of indoloditerpenes, possible biosynthetic intermediates to the tremorgenic mycotoxin, paxilline, from Emericella striata. J. Chem. Soc. Perkin Trans. 1988, 9, 2607-2610. [CrossRef]

24. Kimura, Y.; Nishibe, M.; Nakajima, H.; Hamasaki, T.; Shigemitsu, N.; Sugawara, F.; Stout, T.J.; Clardy, J. Emeniveol: A new pollen growth inhibitor from the fungus, Emericella nivea. Tetrahedr. Lett. 1992, 33, 6987-6990. [CrossRef]

25. Yang, M.H.; Wang, J.S.; Luo, J.G.; Wang, X.B.; Kong, L.Y. Chisopanins A-K, 11 new protolimonoids from chisocheton paniculatus and their anti-inflammatory activities. Bioorg. Med. Chem. 2011, 19, 1409-1417. [CrossRef] [PubMed]

26. Luo, X.D.; Wu, S.H.; Wu, D.G.; Ma, Y.B.; Qi, S.H. Three new apo-tirucallols with six-membered hemiacetal from meliaceae. Tetrahedron 2002, 58, 6691-6695. [CrossRef]

27. Chen, C.; Liang, F.; Chen, B.; Sun, Z.Y.; Xue, T.D.; Yang, R.L.; Luo, D.Q. Identification of demethylincisterol $A_{3}$ as a selective inhibitor of protein tyrosine phosphatase Shp2. Eur. J. Phar. 2016, 795, 124-133. [CrossRef] [PubMed]

28. Sallam, A.A.; Houssen, W.E.; Gissendanner, C.R.; Orabi, K.Y.; Foudah, A.I.; Sayed, K.A.E. Bioguided discovery and pharmacophore modeling of the mycotoxic indole diterpene alkaloids penitrems as breast cancer proliferation, migration, and invasion inhibitors. Med. Chem. Commun. 2013, 4, 1360-1369. [CrossRef] [PubMed]

29. Guo, J.J.; Dai, B.L.; Chen, N.P.; Jin, L.X.; Jiang, F.S.; Ding, Z.S.; Qian, C.D. The anti-staphylococcus aureus activity of the phenanthrene fraction from fibrous roots of Bletilla striata. BMC Complement. Altern. Med. 2016, 16, 491-497. [CrossRef] [PubMed] 